Dear author,

Please note that changes made in the online proofing system will be added to the article before publication but are not reflected in this PDF.

We also ask that this file not be used for submitting corrections. 


\title{
Which creatinine-based estimated glomerular filtration rate equation best predicts all-cause mortality in Chinese subjects with type 2 diabetes?
}

\author{
C.H. Lee ${ }^{a, b}$, A.Z.L. Shih ${ }^{a}$, Y.C. Woo ${ }^{a}$, C.H.Y. Fong ${ }^{a}$, M.M.A. Yuen ${ }^{a}$, W.S. Chow $^{a, *}$, \\ K.S.L. $\operatorname{Lam}^{a, b, c, *}$ \\ ${ }^{a}$ Department of Medicine, The University of Hong Kong, Hong Kong Special Administrative Region \\ ${ }^{\mathrm{b}}$ Research Centre of Heart, Brain, Hormone and Healthy Aging, The University of Hong Kong, Pokfulam, Hong Kong Special Administrative \\ Region \\ c State Key Laboratory of Pharmaceutical Biotechnology, The University of Hong Kong, Pokfulam, Hong Kong Special Administrative Region
}

\section{A R T I C L E I N F O}

Article history:

Received 8 September 2016

Accepted 20 January 2017

Available online $\mathrm{xxxx}$

Keywords:

eGFR equations

Type 2 diabetes

Mortality

Chinese

\begin{abstract}
A B S T R A C T
Aim: In Chinese, ethnicity-based and/or diabetes specific modifications of the Modification of Diet in Renal Disease (MDRD) and the Chronic Kidney Disease Epidemiology Collaboration (CKD-EPI) equations have been developed for determining estimated glomerular filtrate rate (eGFR). This study aimed to compare the performance of five different creatinine-based eGFR equations in predicting all-cause mortality among Chinese subjects with type 2 diabetes (T2DM).

Methods: A total of 6739 Chinese subjects with T2DM were included. Their eGFR was calculated using the MDRD, CKD-EPI, their respective modified equations for Chinese, and the diabetes specific CKD-EPI Chinese T2DM equations. Multiple Cox regression analysis was used to evaluate the associations of eGFR with all-cause mortality. C-statistics, net reclassification index (NRI) and integrated discrimination index (IDI) were applied to assess the discrimination and reclassification of each eGFR equation in predicting mortality outcome. Results: Over a follow-up of 5.7 years, the incidence of all-cause mortality was $12.9 \%$ $(\mathrm{N}=867)$. The CKD-EPI equation discriminated all-cause mortality better than the MDRD equation (C-statistics: 0.714 vs. $0.689, p<0.0001$ ), and Chinese modification of their respective equations did not improve discrimination. Among the five eGFR equations evaluated, the CKD-EPI Chinese T2DM equation provided the best discrimination in predicting allcause mortality among Chinese subjects with T2DM, and was the only equation providing a significantly positive NRI and IDI relative to the CKD-EPI equation.

Conclusions: Among Chinese subjects with T2DM, our findings suggested that the CKD-EPI Chinese T2DM equation best predicted all-cause mortality, and relative to the CKD-EPI equation, conferred improved discrimination and reclassification.
\end{abstract}

(C) 2017 Published by Elsevier Ireland Ltd.

\footnotetext{
* Corresponding authors at: Department of Medicine, University of Hong Kong, Queen Mary Hospital, 102 Pokfulam Road, Pokfulam, Hong Kong Special Administrative Region. Fax: +852 28555411 (W.S. Chow), +852 28162863 (K.S.L. Lam).

E-mail addresses: wschow01@gmail.com (W.S. Chow), ksllam@hku.hk (K.S.L. Lam). http://dx.doi.org/10.1016/j.diabres.2017.01.010
} 0168-8227/@ 2017 Published by Elsevier Ireland Ltd. 


\section{Introduction}

Type 2 diabetes (T2DM) increases mortality. The presence of kidney disease, be it either an impaired glomerular filtration rate (GFR) or albuminuria, further exacerbates this risk through an additive effect [1]. Current clinical guidelines recommend using the Chronic Kidney Disease Epidemiology Collaboration (CKD-EPI) equation over the Modification of Diet in Renal Disease study (MDRD) equation for estimating GFR, owing to the improved accuracy and lower bias with the CKD-EPI equation, especially at higher eGFR levels [2]. In addition, the CKD-EPI equation had been shown to be superior to the MDRD equation in predicting all-cause mortality in Western populations, which in a few studies, also included subjects with T2DM [3-6]. In Chinese, ethnicity-based [7,8] and/ or diabetes-specific modifications [9] of the MDRD and CKDEPI equations have been developed for determining eGFR. Nonetheless, their differential performance in the prediction of mortality risk in T2DM remains largely undefined. Therefore, with a view to address this issue, we performed this study in a large cohort of Chinese subjects with T2DM over a median follow-up of 5.7 years.

\section{Methods}

\subsection{Subjects}

Subjects were recruited from the Hong Kong West Cluster who attended a diabetes complication-screening program between 1st January 2010 and 31st December 2010. Each diabetes complication-screening visit consisted of clinical assessments and laboratory investigations to determine the control of diabetes as well as its related cardiovascular risk factors, and the presence of diabetic complications.

In this study, inclusion criteria comprised being Chinese, aged more than or equal to 18 years old and with serum creatinine data available. Patients who were on renal replacement therapy or had received a kidney transplant were excluded. Therefore, a total of 6739 subjects were eligible and had been followed up for all-cause mortality until 31st January 2016. The study protocol was approved by the Institu- tional Review Board of the University of Hong Kong/Hospital Authority Hong Kong West Cluster.

\subsection{Clinical and biochemical assessments}

Subjects attended each diabetes complication-screening visit 97 after an overnight fast of at least $8 \mathrm{~h}$. Demographic data, including age, gender, smoking and alcohol consumption were obtained. Anthropometric parameters, including body weight, height, body mass index (BMI), waist circumference (WC), and blood pressure (BP) were measured. Fasting blood was drawn for plasma glucose, lipids, glycated hemoglobin (HbA1c) and serum creatinine. eGFR values were determined based on serum creatinine in $\mathrm{mg} / \mathrm{dL}$ and age as at the reference date of serum creatinine measurement, using the MDRD [10], MDRD Chinese [8], CKD-EPI [11], CKD-EPI Chinese [7] and the CKD-EPI Chinese T2DM equations [9]. (Table 1)

\subsection{Definitions of clinical variables and outcomes}

The presence of dyslipidaemia was defined as fasting triglycerides $(\mathrm{TG}) \geqslant 1.69 \mathrm{mmol} / \mathrm{L}$, high density lipoprotein cholesterol (HDL-C) $<1.04 \mathrm{mmol} / \mathrm{L}$ in men and $<1.29 \mathrm{mmol} / \mathrm{L}$ in women, low density lipoprotein cholesterol (LDL-C) $\geqslant$ $3.4 \mathrm{mmol} / \mathrm{L}$ or on lipid-lowering agents. Hypertension was defined as blood pressure $\geqslant 140 / 90 \mathrm{mmHg}$ or on antihypertensive medications. In this study, the outcome of interest, all-cause mortality, was defined as all registered deaths. In this study, all clinical variables, biochemical assessments and outcome of interest were retrieved from the Clinical Data Analysis and Reporting System (CDARS).

\subsection{Statistical analysis}

All data were analyzed with IBM SPSS Statistics 23.0 and R version 3.2.1. Data that were not normally distributed, as determined using Kolmogorov-Smirnov test, were naturallogarithmically transformed to obtain near normality before analysis. Values were reported as means \pm standard deviation (SD), medians with inter-quartile range (IQR), or percentages as appropriate. Multivariable Cox regression analysis was

\begin{tabular}{|c|c|c|}
\hline eGFR equations & Formula & References \\
\hline MDRD Study Equation (MDRD) & $175 \times\left(\mathrm{S}_{\mathrm{Cr}}\right)^{-1.154} \times(\mathrm{age})^{-0.203} \times(0.742$ if female $)$ & [10] \\
\hline $\begin{array}{l}\text { MDRD Chinese Equation } \\
\text { (MDRD Chinese) }\end{array}$ & $175 \times\left(\mathrm{S}_{\mathrm{Cr}}\right)^{-1.154} \times(\mathrm{age})^{-0.203} \times(0.742$ if female $) \times 1.233$ & {$[8]$} \\
\hline $\begin{array}{l}\text { Original CKD-EPI equation } \\
\text { (CKD-EPI) }\end{array}$ & $\begin{array}{l}141 \times\left(\mathrm{S}_{\mathrm{Cr}} / 0.9\right)^{-0.411} \times 0.993^{\text {Age }} \text { if male and serum creatinine } \leqslant 0.9 \mathrm{mg} / \mathrm{dL} \\
141 \times\left(\mathrm{S}_{\mathrm{Cr}} / 0.9\right)^{-1.209} \times 0.993^{\text {Age }} \text { if male and serum creatinine }>0.9 \mathrm{mg} / \mathrm{dL} \\
144 \times\left(\mathrm{S}_{\mathrm{Cr}} / 0.7\right)^{-0.329} \times 0.993^{\text {Age }} \text { if female and serum creatinine } \leqslant 0.7 \mathrm{mg} / \mathrm{dL} \\
144 \times\left(\mathrm{S}_{\mathrm{Cr}} / 0.7\right)^{-1.209} \times 0.993^{\text {Age }} \text { if female and serum creatinine }>0.7 \mathrm{mg} / \mathrm{dL}\end{array}$ & [11] \\
\hline $\begin{array}{l}\text { CKD-EPI Chinese Equation } \\
\text { (CKD-EPI Chinese) }\end{array}$ & $\begin{array}{l}141 \times\left(\mathrm{S}_{\mathrm{Cr}} / 0.9\right)^{-0.411} \times 0.993^{\mathrm{Age}} \times 1.10 \text { if male and serum creatinine } \leqslant 0.9 \mathrm{mg} / \mathrm{dL} \\
141 \times\left(\mathrm{S}_{\mathrm{Cr}} / 0.9\right)^{-1.209} \times 0.993^{\mathrm{Age}} \times 1.10 \text { if male and serum creatinine }>0.9 \mathrm{mg} / \mathrm{dL} \\
144 \times\left(\mathrm{S}_{\mathrm{Cr}} / 0.7\right)^{-0.329} \times 0.993^{\text {Age }} \times 1.10 \text { if female and serum creatinine } \leqslant 0.7 \mathrm{mg} / \mathrm{dL} \\
144 \times\left(\mathrm{S}_{\mathrm{Cr}} / 0.7\right)^{-1.209} \times 0.993^{\text {Age }} \times 1.10 \text { if female and serum creatinine }>0.7 \mathrm{mg} / \mathrm{dL}\end{array}$ & [7] \\
\hline $\begin{array}{l}\text { CKD-EPI Equation Modified for } \\
\text { Type } 2 \text { DM (CKD-EPI Chinese } \\
\text { T2DM) }\end{array}$ & $\begin{array}{l}117 \times\left(\mathrm{S}_{\mathrm{Cr}} / 0.9\right)^{-0.277} \times 0.994^{\text {Age }} \text { if male and serum creatinine } \leqslant 0.9 \mathrm{mg} / \mathrm{dL} \\
102 \times\left(\mathrm{S}_{\mathrm{Cr}} / 0.9\right)^{-0.558} \times 0.994^{\text {Age }} \text { if male and serum creatinine }>0.9 \mathrm{mg} / \mathrm{dL} \\
94 \times\left(\mathrm{S}_{\mathrm{Cr}} / 0.7\right)^{-0.511} \times 0.998^{\text {Age }} \text { if female and serum creatinine } \leqslant 0.7 \mathrm{mg} / \mathrm{dL} \\
128 \times\left(\mathrm{S}_{\mathrm{Cr}} / 0.7\right)^{-0.543} \times 0.992^{\text {Age }} \text { if female and serum creatinine }>0.7 \mathrm{mg} / \mathrm{dL}\end{array}$ & [9] \\
\hline
\end{tabular}


used to evaluate the association between eGFR and all-cause mortality. The discrimination of each creatinine-based eGFR equation in predicting mortality outcome was analysed and compared using $C$ statistics. $C$ statistics from different eGFR equations were compared using CompareC package, $R$ version 3.2.2. The incremental value of each eGFR equations with reference to the currently recommended CKD-EPI equation was assessed by the integrated discrimination improvement (IDI) and the net reclassification index (NRI). In all statistical tests, two-sided $\mathrm{p}$-values $<0.05$ were considered significant.

\section{Results}

A total of 6739 Chinese subjects with T2DM were included in this prospective study. Table 2 summarizes their baseline characteristics. At baseline, $49.7 \%$ were men. Their mean age was $64.6 \pm 12.4$ years with a mean BMI of $25.3 \pm 4.2 \mathrm{~kg} / \mathrm{m}^{2}$. Their mean duration of diabetes was $10.1 \pm 7.9$ years and their mean $\mathrm{HbA1C}$ was $7.5 \pm 1.4 \%$. Among them, $21.3 \%$ were eversmokers, $76.6 \%$ had hypertension, $73.5 \%$ had dyslipidaemia and $12.0 \%$ had prevalent cardiovascular disease at baseline.

Over a median follow-up of $5.7 \pm 1.1$ years, there were 867 deaths (12.9\%). In Cox regression analysis, eGFR determined from all creatinine-based equations was independently associated with all-cause mortality, after adjustments for gender, age, BMI, duration of diabetes, HbA1c, smoking, dyslipidaemia and hypertension (all $p<0.001$; Table 3 ). The hazard ratio (HR) for all-cause mortality was 0.966 for MDRD, 0.966 for CKD-EPI, 0.973 for MDRD Chinese, 0.969 for CKD-EPI Chinese and 0.948 for CKD-EPI Chinese T2DM (Table 3).

The discriminatory and reclassifying abilities of different eGFR equations are shown in Table 4 . In terms of discrimination, in the prediction of all-cause mortality among Chinese

\section{Table 2 - Baseline characteristics of subjects in the study.}

\begin{tabular}{ll} 
Variables & All (N=6739) \\
\hline Male/female (n/n) & $3348 / 3391$ \\
Age, years & $64.6 \pm 12.4$ \\
Duration of diabetes, years & $10.1 \pm 7.9$ \\
HbA1c, \% & $7.5 \pm 1.4$ \\
Ever smokers, \% & 21.3 \\
Hypertension, \% & 76.6 \\
Dyslipidaemia, \% & 73.5 \\
Antihypertensive agents, \% & 67.2 \\
Lipid-lowering agents, \% & 24.1 \\
Insulin therapy, \% & 14.9 \\
Oral hypoglycaemic agents, \% & 62.1 \\
Body mass index, kg/m ${ }^{2}$ & $25.3 \pm 4.2$ \\
SBP, mmHg & $138 \pm 22$ \\
DBP, mmHg & $75 \pm 11$ \\
Total-cholesterol, mmol/L ${ }^{*}$ & $4.6(4.0-5.2)$ \\
LDL-C, mmol/L" & $2.7(2.2-3.2)$ \\
HDL-C, mmol/L & $1.2(1.0-1.4)$ \\
TG, mmol/L ${ }^{*}$ & $1.3(0.9-1.8)$ \\
Prevalent cardiovascular disease, \% & 12.0 \\
\hline Data were presented as means \pm SD or median (IQR); "Data were \\
log-transformed prior to analysis; LDL-C, low-density lipoprotein \\
cholesterol; HDL-C, high-density lipoprotein cholesterol; TG, \\
triglyceride.
\end{tabular}

Table 3 - Cox regression analysis on the association of eGFR with the risk of all-cause mortality.

\begin{tabular}{lll} 
eGFR equations & \multicolumn{2}{l}{ All-cause mortality } \\
\cline { 2 - 3 } & Hazard Ratio & p-value \\
\hline CKD-EPI & & \\
Unadjusted & 0.964 & $<0.001$ \\
Adjusted model 1 & 0.973 & $<0.001$ \\
Adjusted model 2 & 0.966 & $<0.001$ \\
MDRD & & \\
Unadjusted & 0.965 & $<0.001$ \\
Adjusted model 1 & 0.975 & $<0.001$ \\
Adjusted model 2 & 0.966 & $<0.001$ \\
MDRD Chinese & & \\
Unadjusted & 0.971 & $<0.001$ \\
Adjusted model 1 & 0.979 & $<0.001$ \\
Adjusted model 2 & 0.973 & \\
CKD-EPI Chinese & & $<0.001$ \\
Unadjusted & 0.967 & $<0.001$ \\
Adjusted model 1 & 0.976 & $<0.001$ \\
Adjusted model 2 & 0.969 & \\
CKD-EPI Chinese T2DM & & $<0.001$ \\
Unadjusted & 0.942 & $<0.001$ \\
Adjusted model 1 & 0.960 & $<0.001$ \\
Adjusted model 2 & 0.948 & \\
\hline $\begin{array}{l}\text { eGFR, estimated glomerular filtrate rate; Adjusted Model 1: adjus- } \\
\text { ted for gender and age; Adjusted model 2: adjusted for gender, age, } \\
\text { BMI, duration of diabetes, HbA1c, smoking, dyslipidaemia and } \\
\text { hypertension. }\end{array}$ & \\
\hline
\end{tabular}

subjects with T2DM using $C$ statistics, compared with the CKD-EPI equation, both MDRD and its Chinese specific equations discriminated poorly (C statistics: CKD-EPI 0.714 vs. MDRD 0.689, $p<0.0001$; CKD-EPI Chinese 0.714 vs. MDRD Chinese $0.689, p<0.0001)$. The use of Chinese-specific CKD-EPI equation also did not improve discrimination (C statistics of both CKD-EPI and CKD-EPI Chinese $=0.714$ ), whereas the Chinese and diabetes specific CKD-EPI Chinese T2DM had the best discrimination ( $C$ statistics: CKD-EPI Chinese T2DM 0.724 vs. CKD-EPI Chinese $0.714, p<0.0001)$. Compared with the CKD-EPI equation, the IDI analysis also showed a modest but significant improvement in predicting all-cause mortality using the CKD-EPI Chinese T2DM (IDI +0.757, $p<0.0001$ ), while it was the reverse for MDRD, MDRD Chinese and CKDEPI Chinese equations. With regard to reclassification, similarly, when compared with the CKD-EPI equation, both the MDRD and its Chinese-specific equations resulted in a significant decrease in the NRI for predicting all-cause mortality. The use of CKD-EPI Chinese equation also resulted in a mild, although insignificant, decrease in the NRI. On the contrary, the CKD-EPI Chinese T2DM provided a significant increase in the NRI for all-cause mortality (NRI +5.595, $p<0.0001$ ). Taken together, among Chinese patients with T2DM, the CKD-EPI Chinese T2DM best predicted all-cause mortality with the highest $\mathrm{C}$-index, and was the only equation that significantly improved both the IDI and NRI for all-cause mortality relative to the currently recommended CKD-EPI equation. 
Table 4 - Discrimination and reclassification performance of different eGFR equations in predicting all-cause mortality, based on C-statistics, net reclassification index (NRI) and integrated discrimination improvement (IDI).

\begin{tabular}{llllllll} 
eGFR equations & C-statistics, (SE) & $\begin{array}{l}\text { Difference of } \\
\text { C-statistics }\end{array}$ & $p$-Value & NRI, \% (SE) & $p$-Value IDI, \% (SE) & $p$-Value \\
\hline CKD-EPI & $0.714(0.961)$ & Referent & - & Referent & - & Referent & - \\
MDRD & $0.689(1.030)$ & -0.025 & $<0.0001$ & $-1.456(0.395)$ & 0.0002 & $-2.264(0.089)$ & $<0.0001$ \\
MDRD Chinese & $0.689(1.030)$ & -0.025 & $<0.0001$ & $-9.513(1.671)$ & $<0.0001$ & $-2.333(0.093)$ & $<0.0001$ \\
CKD-EPI Chinese & $0.714(0.961)$ & 0.00 & Not applicable & $-1.700(1.057)$ & 0.1079 & $-0.283(0.018)$ & $<0.0001$ \\
CKD-EPI Chinese T2DM & $0.724(0.988)$ & +0.01 & $<0.0001$ & $+5.595(1.168)$ & $<0.0001$ & $+0.757(0.120)$ & $<0.0001$ \\
\hline
\end{tabular}

\section{Discussions}

To our knowledge, this is the first study comparing the performance of different creatinine-based eGFR equations in predicting all-cause mortality in Chinese subjects with T2DM. In this regard, our results suggested that the CKD-EPI Chinese T2DM equation appeared to perform the best, in terms of both satisfactory discrimination and reclassification. In keeping with the current clinical recommendations, we demonstrated that the CKD-EPI equation, be it the original or the Chinese modified version, was superior to the MDRD study equation or its Chinese version, not only in terms of GFR determination, but also in the prediction of all-cause mortality and cardiovascular mortality, as previously demonstrated in Western populations [3-6]. A meta-analysis involving 1.1 million adults from 25 general population cohorts, 7 cohorts of subjects at high cardiovascular risk, and 13 cohorts of subjects with chronic kidney disease (CKD), had shown that the CKD-EPI equation improved reclassification with respect to risks of both all-cause and cardiovascular mortality, compared with the MDRD equation. Moreover, NRI was consistently positive for mortality outcomes irrespective of the presence or absence of diabetes [4]. Similarly, in another study involving 2823 subjects with T2DM, the areas under the receiving operating characteristic curves (AUROCs) for all-cause mortality and cardiovascular mortality were significantly higher with GFR estimated from the CKD-EPI equation, than those from the MDRD equation, highlighting the more accurate risk stratification of the CKD-EPI equation [6]. Bias in GFR estimation could result in misdiagnosis of CKD, and influences risks of CKD-related complications including allcause and cardiovascular mortality. Therefore, the CKD-EPI equation, with less bias than the MDRD equation, especially at higher levels of eGFR [11], should theoretically also have increased accuracy in predicting mortality outcome.

In our study, ethnicity-based modification did not improve mortality prediction in both the CKD-EPI and MDRD equations. In a multiethnic study comprising $40.5 \%$ Chinese, it had been shown that the CKD-EPI equation was less affected by ethnicity than the MDRD Study equation and even suggested that ethnic adjustments might not be truly necessary when using the CKD-EPI equation, given the small average difference in GFR estimation [7]. In another study performed in the Chinese population, the addition of ethnic coefficient also did not improve GFR estimation [12].

Among the five creatinine-based equations assessed in this study, the CKD-EPI Chinese T2DM equation appeared to provide the best discrimination and, relative to the CKD-EPI, conferred satisfactory reclassification in predicting all-cause mortality in our cohort. Our data would suggest that this disease-specific equation for GFR estimation might be more helpful in stratifying mortality risk among Chinese subjects with T2DM. Indeed, differences in body mass index, muscle mass as well as glycaemic status could influence the estimation of GFR $[9,13]$. A cross-sectional study done in Brazil had previously shown that both the CKD-EPI and MDRD equations could result in significant under-estimation of measured GFR among 105 subjects with T2DM, especially at higher GFR levels [14]. Notably, in the development and validation dataset of the original CKD-EPI equation, only around a third of subjects had diabetes with only $1-2 \%$ being Asians [11]. Therefore, compared with the original CKD-EPI equation, the CKD-EPI Chinese T2DM equation appeared to achieve less bias and a higher precision in GFR estimation among Chinese subjects with T2DM [9].

The strength of our study is the prospective, observational design with a large representative sample of Chinese subjects with T2DM. However, an important limitation is the relatively low number of events that could have influenced the comparison of the performance of different eGFR equations in predicting this mortality outcome. Moreover, our results might not be generalizable to patients with more severe CKD stages as more than $70 \%$ of our subjects had eGFR $>60 \mathrm{ml} /$ $\min / 1.73 \mathrm{~m}^{2}$ with relatively preserved kidney function. Finally, since direct GFR measurements are not available in this study, a gold standard is not available for a comprehensive comparison of various eGFR equations to predict mortality outcomes.

Notwithstanding these limitations, our results demonstrated that, first, in keeping with current recommendations; the CKD-EPI equation is superior to the MDRD equation for the prediction of all-cause mortality. Second, compared with the currently recommended CKD-EPI equation, this diabetes-specific CKD-EPI Chinese T2DM equation appears to improve the performance in predicting all-cause mortality among Chinese subjects with T2DM, especially in those with relatively preserved kidney functions. Further prospective studies, using a larger cohort with longer duration of followup, are warranted to validate our findings on the importance of disease-specificity in the use of the CKD-EPI equation for both GFR estimation and mortality prediction in patients with T2DM.

\section{Disclosures}

All authors declare no conflicts of interest. 


\section{Author contributions}

C.H.L. researched the data and wrote the manuscript. A.Z.L.S, Y.C.W. and M.M.A.Y. researched the data. A.Z.L.S. and C.H.Y.F. performed statistical analyses. W.S.C. and K.S.L.L. initiated and supervised the study, critically reviewed and edited the manuscript, and are the guarantors of this work and as such had full access to all the data in the study and take responsibility for the integrity of the data and the accuracy of the data analysis.

R E F E R E N C E S

[1] Afkarian M, Sachs MC, Kestenbaum B, Hirsch IB, Tuttle KR, Himmelfarb J, et al. Kidney disease and increased mortality risk in type 2 diabetes. J Am Soc Nephrol: JASN 2013;24:302-8.

[2] Chapter 2: Definition, identification, and prediction of CKD progression. Kidney Int Suppl. 2013:63-72.

[3] Shafi T, Matsushita K, Selvin E, Sang Y, Astor BC, Inker LA, et al. Comparing the association of GFR estimated by the CKD-EPI and MDRD study equations and mortality: the third national health and nutrition examination survey (NHANES III). BMC Nephrol. 2012;13:42.

[4] Matsushita K, Mahmoodi BK, Woodward M, Emberson JR, Jafar $\mathrm{TH}$, Jee $\mathrm{SH}$, et al. Comparison of risk prediction using the CKD-EPI equation and the MDRD study equation for estimated glomerular filtration rate. JAMA 2012;307:1941-51.

[5] White SL, Polkinghorne KR, Atkins RC, Chadban SJ. Comparison of the prevalence and mortality risk of CKD in Australia using the CKD Epidemiology Collaboration (CKDEPI) and Modification of Diet in Renal Disease (MDRD) Study GFR estimating equations: the AusDiab (Australian Diabetes, Obesity and Lifestyle) Study. Am J Kidney Dis 2010;55:660-70.
[6] Targher G, Zoppini G, Mantovani W, Chonchol M, Negri C, Stoico V, et al. Comparison of two creatinine-based estimating equations in predicting all-cause and cardiovascular mortality in patients with type 2 diabetes. Diabetes Care 2012;35:2347-53.

[7] Teo BW, Xu H, Wang D, Li J, Sinha AK, Shuter B, et al. GFR estimating equations in a multiethnic Asian population. Am J Kidney Dis 2011;58:56-63.

[8] Ma YC, Zuo L, Chen JH, Luo Q, Yu XQ, Li Y, et al. Modified glomerular filtration rate estimating equation for Chinese patients with chronic kidney disease. J Am Soc Nephrol 2006;17:2937-44.

[9] Liu X, Gan X, Chen J, Lv L, Li M, Lou T. A new modified CKDEPI equation for Chinese patients with type 2 diabetes. PLoS ONE 2014;9:e109743.

[10] Levey AS, Bosch JP, Lewis JB, Greene T, Rogers N, Roth D. A more accurate method to estimate glomerular filtration rate from serum creatinine: a new prediction equation. Ann Internal Med 1999;130:461-70.

[11] Levey AS, Stevens LA, Schmid CH, Zhang YL, Castro 3rd AF, Feldman HI, et al. A new equation to estimate glomerular filtration rate. Ann Intern Med 2009;150:604-12.

[12] Kong X, Ma Y, Chen J, Luo Q, Yu X, Li Y, et al. Evaluation of the Chronic Kidney Disease Epidemiology Collaboration equation for estimating glomerular filtration rate in the Chinese population. Nephrol Dial Transplant 2013;28:641-51.

[13] Kawamoto R, Kohara K, Tabara Y, Miki T, Ohtsuka N, Kusunoki T, et al. An association between body mass index and estimated glomerular filtration rate. Hypertens Res 2008;31:1559-64.

[14] Silveiro SP, Araujo GN, Ferreira MN, Souza FD, Yamaguchi HM, Camargo EG. Chronic Kidney Disease Epidemiology Collaboration (CKD-EPI) equation pronouncedly underestimates glomerular filtration rate in type 2 diabetes. Diabetes Care 2011;34:2353-5. 